\title{
Alteration of metabolite profiling by cold atmospheric plasma treatment in human myeloma cells
}

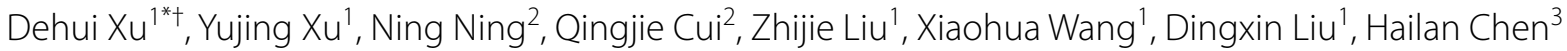
and Michael G. Kong ${ }^{1,3,4^{*}+}$

\begin{abstract}
Background: Despite new progress of chemotherapy in multiple myeloma (MM) clinical treatment, MM is still a refractory disease and new technology is needed to improve the outcomes and prolong the survival. Cold atmospheric plasma is a rapidly developed technology in recent years, which has been widely applied in biomedicine. Although plasma could efficiently inactivate various tumor cells, the effects of plasma on tumor cell metabolism have not been studied yet.

Methods: In this study, we investigated the metabolite profiling of He plasma treatment on myeloma tumor cells by gas-chromatography time-of-flight (GC-TOF) mass-spectrometry. Meanwhile, by bioinformatic analysis such as GO and KEGG analysis we try to figure out the metabolism pathway that was significantly affected by gas plasma treatment.
\end{abstract}

Results: By GC-TOF mass-spectrometry, 573 signals were detected and evaluated using PCA and OPLS-DA. By KEGG analysis we listed all the differential metabolites and further classified into different metabolic pathways. The results showed that beta-alanine metabolism pathway was the most significant change after He gas plasma treatment in myeloma cells. Besides, propanoate metabolism and linoleic acid metabolism should also be concerned during gas plasma treatment of cancer cells.

Conclusions: Cold atmospheric plasma treatment could significantly alter the metabolite profiling of myeloma tumor cells, among which, the beta-alanine metabolism pathway is the most susceptible to He gas plasma treatment.

Keywords: Cold atmospheric plasma, Multiple myeloma, Metabolite profiling, Mass spectrometry, KEGG analysis, Beta-Alanine metabolism

\section{Background}

Multiple myeloma (MM) is a malignant tumor caused by abnormal proliferation of monoclonal plasma cells, accounting for $1 \%$ of all tumors and $13 \%$ of hematological malignancies $[1,2]$. After the initial onset of remission, relapse will occur and only $25 \%$ of patients have a survival of more than 5 years after receiving chemotherapy

\footnotetext{
*Correspondence: dehuixu@hotmail.com; mkong@odu.edu

${ }^{\dagger}$ Dehui Xu and Michael G. Kong contributed equally to this work

${ }^{1}$ State Key Laboratory of Electrical Insulation and Power Equipment,

Centre for Plasma Biomedicine, Xi'an Jiaotong University, Xi'an 710049

Shaanxi, People's Republic of China

Full list of author information is available at the end of the article
}

[3]. It is characterized by an increase in abnormal plasma cells that produce monoclonal immunoglobulin and malignant proliferation in the bone marrow, causing fractures and bone marrow failure $[4,5]$. The current clinical treatment of MM includes radiation therapy, bone marrow transplantation and chemotherapeutics treatment $[6,7]$. Radiation therapy, however, will inevitably damage human normal cells while killing cancer cells. Bone marrow transplantation may result in postoperative autologous rejection. Chemotherapeutics may have serious side effects and usually lead to drug resistance. Therefore, MM is a refractory disease and new technology and treatment tools need to be developed for MM therapy. 
Cold atmospheric plasma (CAP) is a new technology rapidly developed in recent years. It is produced under atmospheric pressure with low gas temperature and high activity of reactive species, which has aroused widespread concern especially in biomedical application, such as disinfection of bacteria, application in dermatology and dentistry, cell transfection, wound healing and cancer treatment [8-17]. It is widely reported that plasma could efficiently inactive tumor cells in various types of cancer, including lung cancer, leukemia, intestinal cancer, melanoma, cervical cancer, glioma, multiple myeloma, pancreatic cancer et al. [18-28]. The induction of apoptosis in cancer cells has been widely reported, and the mechanism of plasma-induced apoptosis is being increasingly understood. However, the effects of plasma on tumor cell metabolism have not been reported yet. Cell metabolism is a general term for a series of ordered chemical reactions that take place in the cells to survive. These reaction processes allow cells to grow and reproduce, maintain their functions and respond to the external environment, including the metabolism of matter and energy. Tumor cells provide a source of their aberrant proliferation through a systematic reprogramming of cellular metabolism $[29,30]$. These changes in metabolism involve the production of energy required for cell division, the regulation of intracellular redox status, and the breakdown and synthesis of nutrients after ingestion, thereby altering the flux of metabolites inside and outside cells and redistributing them to the corresponding metabolic pathways, to meet the needs of maintaining the malignant transformation phenotype of cells [31]. Therefore, understanding the effects of gas plasma on tumor cell metabolism is of great significance. In this study, we explored the influence of gas plasma on tumor cell metabolism profiling for the first time. By metabonomics, we found that the metabolism of myeloma tumor cells was greatly changed after He plasma treatment. Notably, beta-alanine metabolism pathway was found to be the major target that was affected by gas plasma treatment, indicating that beta-alanine might play an important role in the interaction of gas plasma with tumor cells.

\section{Methods}

\section{Gas plasma generation}

In this study, we used a plasma jet which was described in our previous research to generate the cold atmospheric plasma. Characters of the plasma generation and electronic parameters were illustrated in our previous works $[17,28,32]$. The He plasma was generated at $10 \mathrm{kHz} / 8 \mathrm{kV}$ with a He gas flow of 2 SLM.

\section{Cell culture condition}

The LP-1 multiple myeloma cell line was used in this study. LP-1 cells were grown in Roswell Park Memorial Institute (RPMI) 1640 medium supplemented with $10 \%$ fetal calf serum, $100 \mathrm{U} / \mathrm{mL}$ penicillin, and $50 \mu \mathrm{g} / \mathrm{mL}$ streptomycin (Gibco-Invitrogen, Carlsbad, CA, 15140122). The cells were cultured at $37{ }^{\circ} \mathrm{C}$ in an incubator (Thermo Scientific, Waltham, MA, USA) containing 5\% $\mathrm{CO}_{2}$. The medium was refreshed $24 \mathrm{~h}$ before performing experiments.

\section{Cell viability assessment}

Cell viability was measured by A Cell-Titer-Glo ${ }^{\circledR}$ luminescent cell viability assay kit (Promega, Madison, WI, USA) which based on the production of ATP in viable cells. $100 \mu \mathrm{L}$ of samples and $100 \mu \mathrm{L}$ of Cell-Titer-Glo ${ }^{\circledR}$ reagent were added to the opaque-walled plate and was incubated at room temperature for $10 \mathrm{~min}$. The luminescence was detected by a microplate reader (Thermo Scientific Varioskan Flash, Waltham, MA, USA) with the protocol of "luminometric" measurement.

\section{Solvents and reagents}

We bought L-2-chlorophenylalanine from Hengbai Biotechnology Co Ltd (Shanghai, China), while Methoxy amination hydrochloride (chromatographic grade), pyridine and chloroform (HPLC grade) were from Admas (Shanghai, China). Moreover, BSTFA (including 1\% TMCS, v/v) was purchased from REGIS Technologies Inc (Morton Grove, IL, USA) and methyl alcohol (HPLC grade) was purchased from ANPEL Laboratory Technologies Inc (Shanghai, China). Saturated fatty acid methyl fat (C8, C9, C10, C12, C14, C16, C18, C20, C22, C24) was bought from Dr. Ehrenstorfer (Augsburg, Germany). Deionized water was used throughout this experiment (Thermo; Waltham, MA, USA).

\section{Sample collection}

$3 \times 10^{5}$ cells/well in $300 \mu \mathrm{L}$ of medium were seeded in 24-well plate, then the wells which treated with $40 \mathrm{~s}$ of $\mathrm{He}$ plasma were considered as plasma treatment group and the remains were control group, and each group had 5 duplicates/samples. After $24 \mathrm{~h}$ incubation, cells were collected and counted to ensure the cell number was about $1 \times 10^{7}$ cells/sample. Cells were harvested by centrifugation at $4{ }^{\circ} \mathrm{C}$ for $5 \mathrm{~min}$ with the speed of $1200 \mathrm{rpm}$ and washed with PBS three times at $4{ }^{\circ} \mathrm{C}$ for 3 min with the speed of $900 \mathrm{rpm}$. Then the cell pellet in EP tube was rapidly put in liquid nitrogen for $5 \mathrm{~min}$ and stored in $-80^{\circ} \mathrm{C}$ refrigerator until analysis. 


\section{Sample preparation}

Before metabolite analysis, sample was mixed with $0.6 \mathrm{~mL}$ of extraction liquid $\left(\mathrm{V}_{\text {methanol }}: \mathrm{V}_{\text {Chlorofrom }}=3: 1\right)$ in $2 \mathrm{~mL}$ EP tube and $10 \mu \mathrm{L}$ of L-2-chlorophenylalanine $\left(1 \mathrm{mg} / \mathrm{mL}\right.$ stock in $\mathrm{dH}_{2} \mathrm{O}$ ) which was regarded as internal standard. After $30 \mathrm{~s}$ of vortex mixing, steel balls were added and grinded for $4 \mathrm{~min}$ at $45 \mathrm{~Hz}$ followed by treating with ultrasound for $5 \mathrm{~min}$ in ice water, then repeating this step for 3 times. The supernatant $(0.5 \mathrm{~mL})$ was transferred into a fresh $2 \mathrm{~mL} \mathrm{GC/MS}$ glass vial after centrifuging for $15 \mathrm{~min}$ at $13,000 \mathrm{rpm}, 4{ }^{\circ} \mathrm{C}$. Next, the extracted metabolites were dried in a vacuum concentrator without heating and $30 \mu \mathrm{L}$ of methoxy amination hydrochloride was added. After incubating in oven at $80{ }^{\circ} \mathrm{C}$ for $30 \mathrm{~min}$, $40 \mu \mathrm{L}$ of the BSTFA regent (1\% TMCS, v/v) was mixed well with the sample aliquots and cultured for $1.5 \mathrm{~h}$ for $70{ }^{\circ} \mathrm{C}$ to obtain the derived metabolites for $\mathrm{GC}-\mathrm{MS}$ analysis.

\section{GC-TOFMS analysis}

GC-TOFMS analysis was performed using an Agilent 7890 gas chromatograph system coupled with a Pegasus HT time-of-flight mass spectrometer. The system utilized a DB-5MS capillary column coated with $5 \%$ diphenyl cross-linked with $95 \%$ dimethylpolysiloxane $(30 \mathrm{~m} \times 250 \mu \mathrm{m}$ inner diameter, $0.25 \mu \mathrm{m}$ film thickness; J\&W Scientific, Folsom, CA, USA). A $1 \mu \mathrm{L}$ aliquot of the analyte was injected in splitless mode. Helium was used as the carrier gas, the front inlet purge flow was $3 \mathrm{~mL} \mathrm{~min}^{-1}$, and the gas flow rate through the column was $1 \mathrm{~mL} \mathrm{~min}^{-1}$. The initial temperature was kept at $80{ }^{\circ} \mathrm{C}$ for $1 \mathrm{~min}$, then raised to $290{ }^{\circ} \mathrm{C}$ at a rate of $10{ }^{\circ} \mathrm{C} \mathrm{min}{ }^{-1}$, then kept for $12 \mathrm{~min}$ at $290{ }^{\circ} \mathrm{C}$. The injection, transfer line, and ion source temperatures were 280 , 295 , and $220{ }^{\circ} \mathrm{C}$, respectively. The energy was $-70 \mathrm{eV}$ in electron impact mode. The mass spectrometry data were acquired in full-scan mode with the $m / z$ range of 50-600 at a rate of 12.02 spectra per second after a solvent delay of $8.45 \mathrm{~min}$.

\section{Results \\ Metabolic profiling of gas plasma-treated cells samples by GC-TOF}

We investigated a total of 12 samples. Six were LP-1 cells with gas flow as control group, another six samples were LP-1 cells treated with He plasma for $1 \mathrm{~min}$. With gas chromatography-time of flight mass spectrometry (GCTOF), around 573 signals were detected per sample using mass spectral deconvolution software for peak detection. However, some of these signals were not consistently found in other samples or were of too low abundance or too poor spectral quality to be unambiguously assigned to unique metabolites. We have normalized with internal standard (IS) and finally 561 valid peaks were remained for further analysis. Details of these 561 peaks were listed in Additional file 1: Table S1. Figure 1 shows the overall representative GC-TOF chromatograms of control group and plasma treatment group.

\section{Unsupervised evaluation of metabolite signatures using PCA and OPLS-DA}

After obtaining the raw data, we carry out a series of multivariate variable pattern recognition analysis, which is the principal component analysis (PCA). Using the SIMCA software (V14.1, MKS Data Analytics Solutions, Umea, Sweden), the data is logarithmic (LOG) formatted (CTR) formatted and then automatically modeled [6]. The parameters of the PCA model are shown in the statistical model parameter Table 1. Since the two groups of samples are not very significant, the sample is basically in the 95\% confidence interval (Hotelling's T-squared ellipse), this data need to be further analyzed.

Therefore, we use the orthogonal least squares-discriminant analysis (orthogonal projection to latent structures-discriminant analysis, OPLS-DA) statistical methods to further analyze the results. Through the OPLS-DA analysis, we can filter out the orthogonal variables that are not related to the categorical variables in the metabolites, and analyze the differences between the non-orthogonal and orthogonal variables to obtain the more reliable metabolites. LOGG software was added to the data using SIMCA software (V14.1, MKS Data Analytics Solutions, Umea, Sweden). First, the first principal component was analyzed by OPLS-DA model. The quality of the model was verified by sevenfold cross validation (model-to-class variability Y) and Q2 (predictability of the model) to determine the validity of the model by cross validation. Finally, Permutation test), randomly different times to change the order of the variable $\mathrm{Y}$ order to get a different random Q2 value, the model validity for further testing. For more information on the OPLS-DA model, refer to the statistical model parameter Table 2 .

As shown in Fig. 2a, the abscissa t [1] P represents the predicted principal component score of the first principal component, and the ordinate $t$ [1] O represents the orthogonal principal component score, and the scatter shape and color represent different experimental groups. It shows that the two groups of samples are more significant, the sample is basically in the $95 \%$ confidence interval (Hotelling's T-squared ellipse). The permutation test establishes the corresponding OPLS-DA model to obtain the random model R2 and Q2 values by randomly changing the permutation order of the categorical variable $\mathrm{Y}$, the number of times $(n=200)$, the avoidance of the overfitting of the test model and the evaluation of the model 

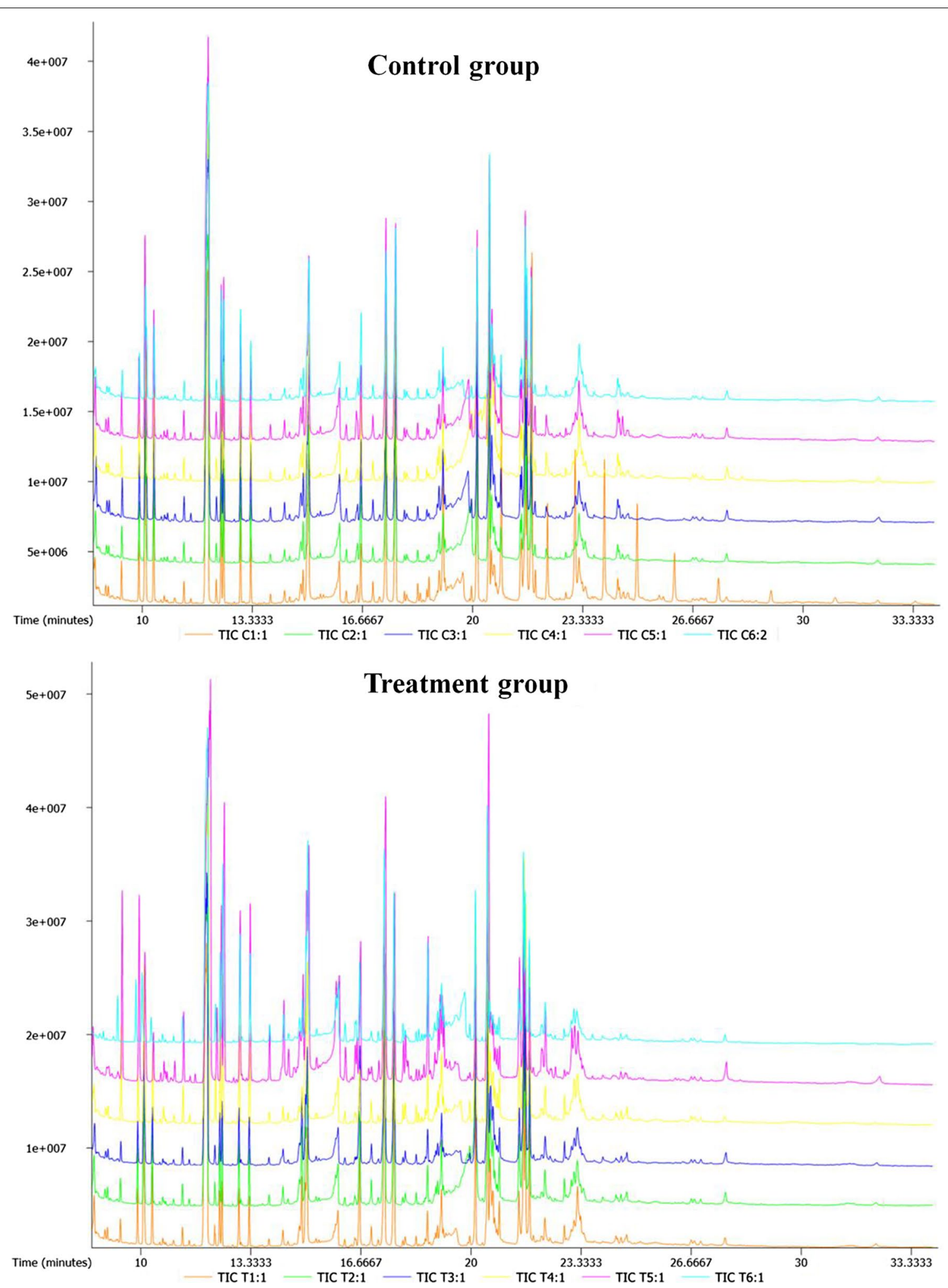

Fig. 1 Representative GC-TOF chromatograms of control group and plasma treatment group by mass spectra 
Table 1 Statistical model parameters table of PCA model

\begin{tabular}{lllll}
\hline Model & Type & $\mathbf{A}$ & $\mathbf{N}$ & $\mathbf{R}^{\mathbf{2}} \mathbf{X}$ (cum) \\
\hline Model 1 & PCA & 3 & 12 & 0.569 \\
\hline
\end{tabular}

Significance has an important role. Figure $2 \mathrm{~b}$ shows the replacement test for the OPLS-DA model.

\section{Identification of differential metabolites by supervised analysis}

We used the generally recognized standard by academia, that is $\mathrm{P}$ value of student's $\mathrm{t}$ test is less than 0.05 , and the variable importance in the projection (VIP) of the first principal component of OPLS-DA model is greater than 1 , to determine the differential metabolites between control and plasma treatment group. Additional file 1: Table S1 lists all the differences in metabolite screening, and was further illustrated in the form of volcano plot (Fig. 3). As shown in the final screening result, the significant uptake of the metabolites was displayed in red, while the significant down-regulation of the metabolites was shown in blue.

\section{KEGG analysis of differential metabolites}

The complex metabolic responses and their regulation in organisms are not carried out separately, and often complex pathways and networks are formed by different genes and proteins. Their interaction and mutual regulation eventually lead to systemic changes in the metabolic group. The analysis of these metabolic and regulatory pathways can provide a more comprehensive and systematic understanding of the biological processes such as changes in the biological processes, the pathogenesis of the disease or the mechanism of the drug.

The kyoto encyclopedia of genes and genomes (KEGG) pathway database (http://www.kegg.jp/kegg/pathway. html) is based on the functional information of genes and genomes, and the metabolic response is clues, the possible metabolic pathways and the corresponding regulatory proteins, in a graphical way to show the cell physiological and biochemical processes. First, we mapped all the 561 metabolites to the Homo sapiens in the KEGG PATHWAY database. The mapping results are shown in Additional file 1.

Based on the mapping results, we sort out all the pathways for the differential metabolite mapping, as shown in Additional file 2. After that, we labeled the differential metabolites on the KEGG pathway. As shown in Fig. 4, red represented up-regulation while bright blue represented down-regulation. And the black indicated that metabolites were detected but not significantly different.

\section{Metabolic pathway analysis of differential metabolites}

KEGG analysis only found all the pathways that was relevant to these differential metabolites, to further analyze the most relevant pathways associated with these metabolite differences, a comprehensive analysis of the pathways of differential metabolites (including enrichment analysis and topological analysis) was carried out. We mapped these differential metabolites to the metabolites database such as KEGG, PubChem et al. and the metabolite mapping table was shown in Additional file 3. After obtaining the matching information of the different metabolites, we searched and analyzed the metabolic pathways in Homo sapiens database. An example of a metabolic pathway analysis table is given in Additional file 4 . The results of the metabolic pathway analysis were presented as a bubble chart (Fig. 5). Each bubble in the bubble diagram represents a metabolic pathway. The abscissa and bubble size of the bubble indicate the size of the influence factor in the topology analysis. The larger the size is, the greater the influence factor is. The vertical and bubble color of the bubble indicate the enrichment analysis $\mathrm{P}$ value (negative natural logarithm, that is-ln $\mathrm{P}$ value), the deeper the color $\mathrm{P}$ value is smaller, the more significant degree of enrichment. From this chart we could clear figure out that beta-alanine metabolism pathway was the most significant changes after He gas plasma treatment in LP-1 cells. Besides, propanoate metabolism and linoleic acid metabolism should also be concerned during gas plasma treatment of cancer cells.

\section{Hierarchical clustering analysis of differential metabolites}

The differential metabolites obtained by the above analysis are often biologically consistent and functional similarity/complementarity, or positive regulation/negative regulation by the same metabolic pathway, which will present similar or opposite expression characteristics between different experimental groups. The hierarchical clustering analysis of these characteristics will clear classify the metabolites with the same and different characteristics between the experimental groups.

We calculated the Euclidean distance matrix from the plasma treatment group to quantify the differential metabolites of the control group. The results were

Table 2 Statistical model parameters table of OPLS-DA model

\begin{tabular}{llllll}
\hline Model & Type & $\mathbf{A}$ & $\mathbf{N}$ & $\mathbf{R}^{\mathbf{2} X(\text { cum })}$ & $\mathbf{R}^{\mathbf{2}}$ (cum) \\
\hline Model 2 & OPLS-DA & $1+1+0$ & 12 & 0.486 & 0.985 \\
\hline
\end{tabular}




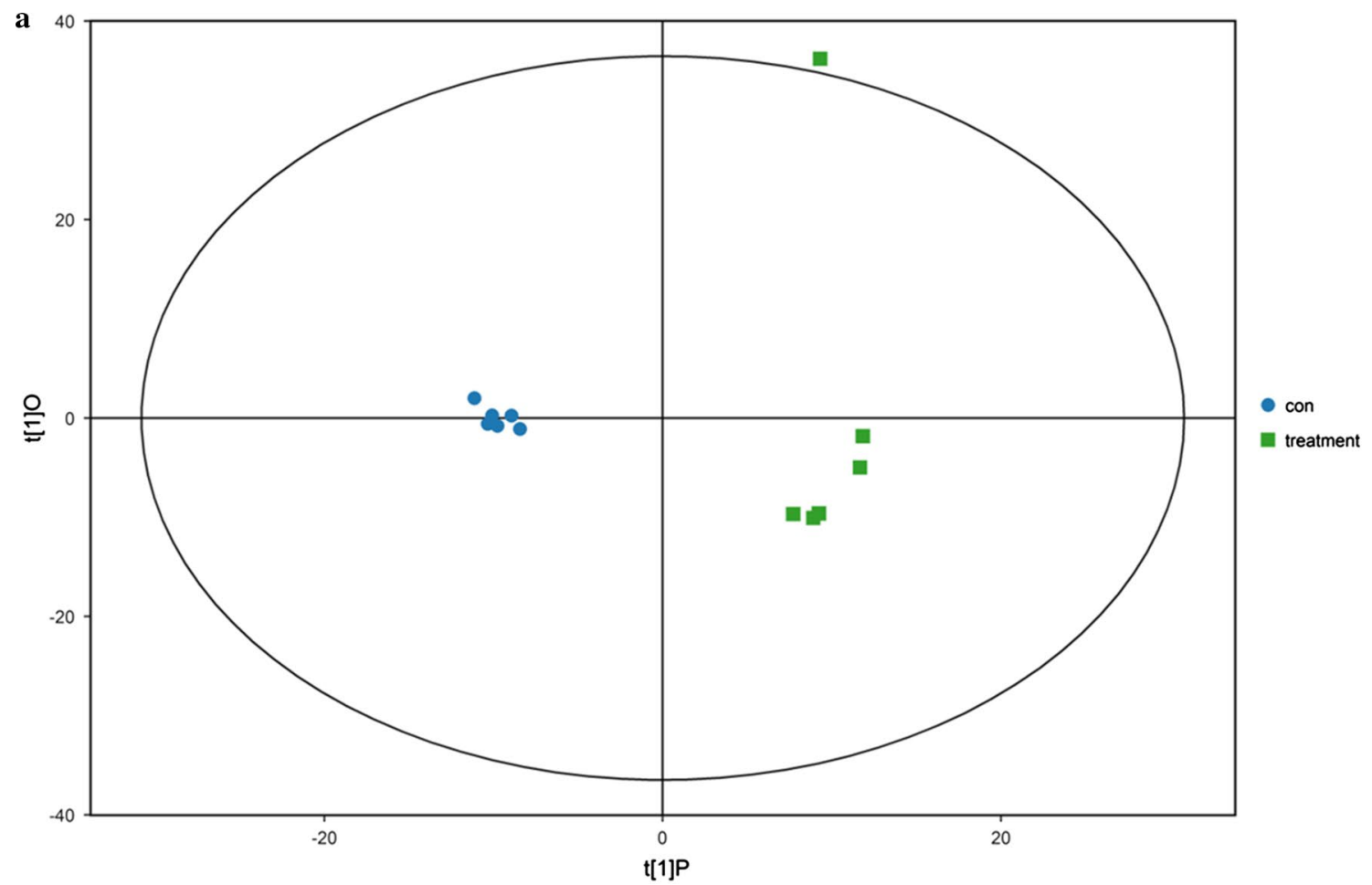

b

Intercepts: $R^{2} Y($ cum $)=(0,0.8), Q^{2}($ cum $)=(0,-0.61)$

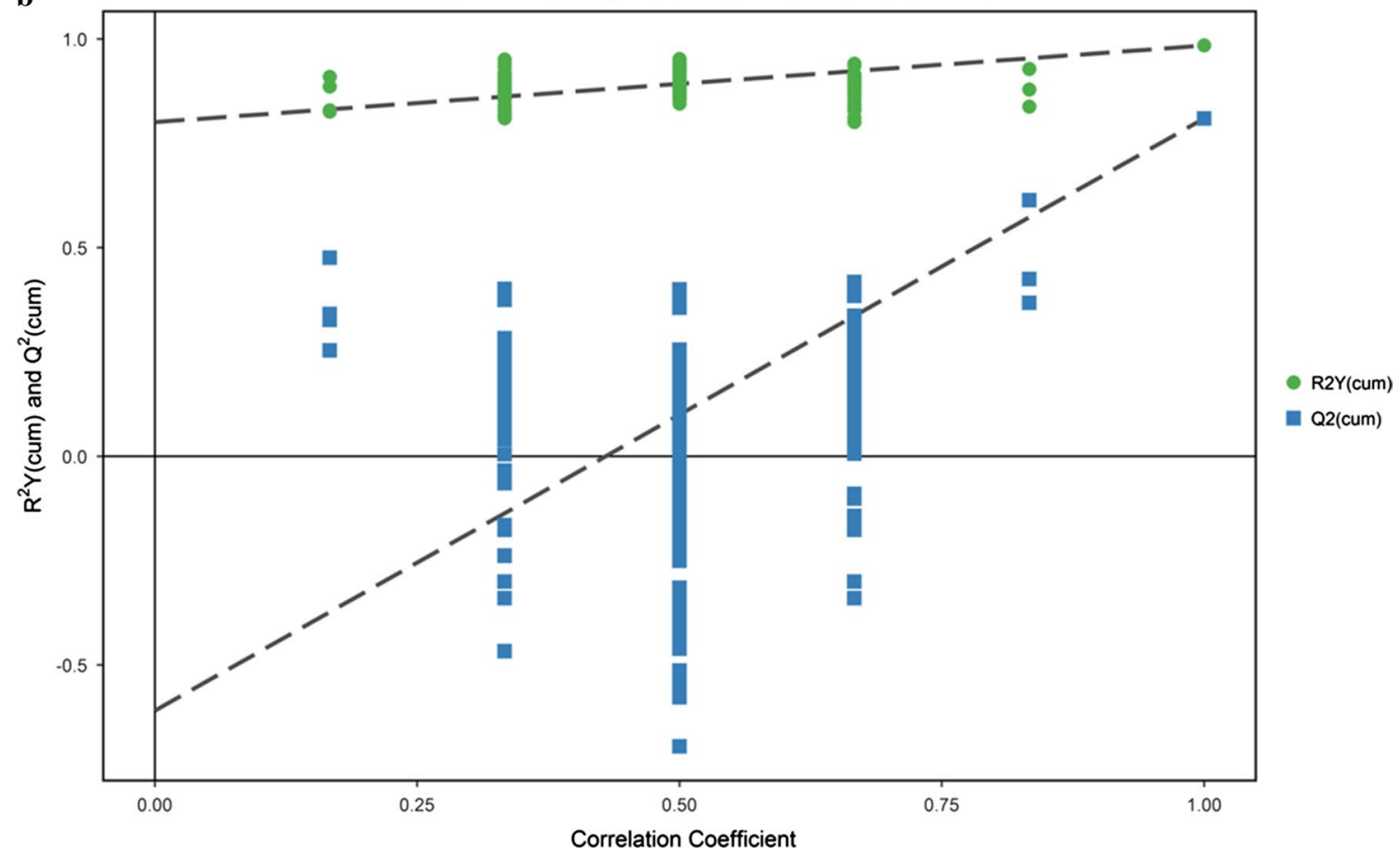

Fig. 2 The score scatter plot of (a) OPLS-DA model and permutation test of (b) OPLS-DA model

visualized in a heatmap that was combined with hierarchical clustering of samples and metabolites (Fig. 6). The color patches at different positions represent the relative expression levels of the corresponding metabolites. It can be seen that there are obvious differences in metabolic grouping patterns after He plasma treatment. 


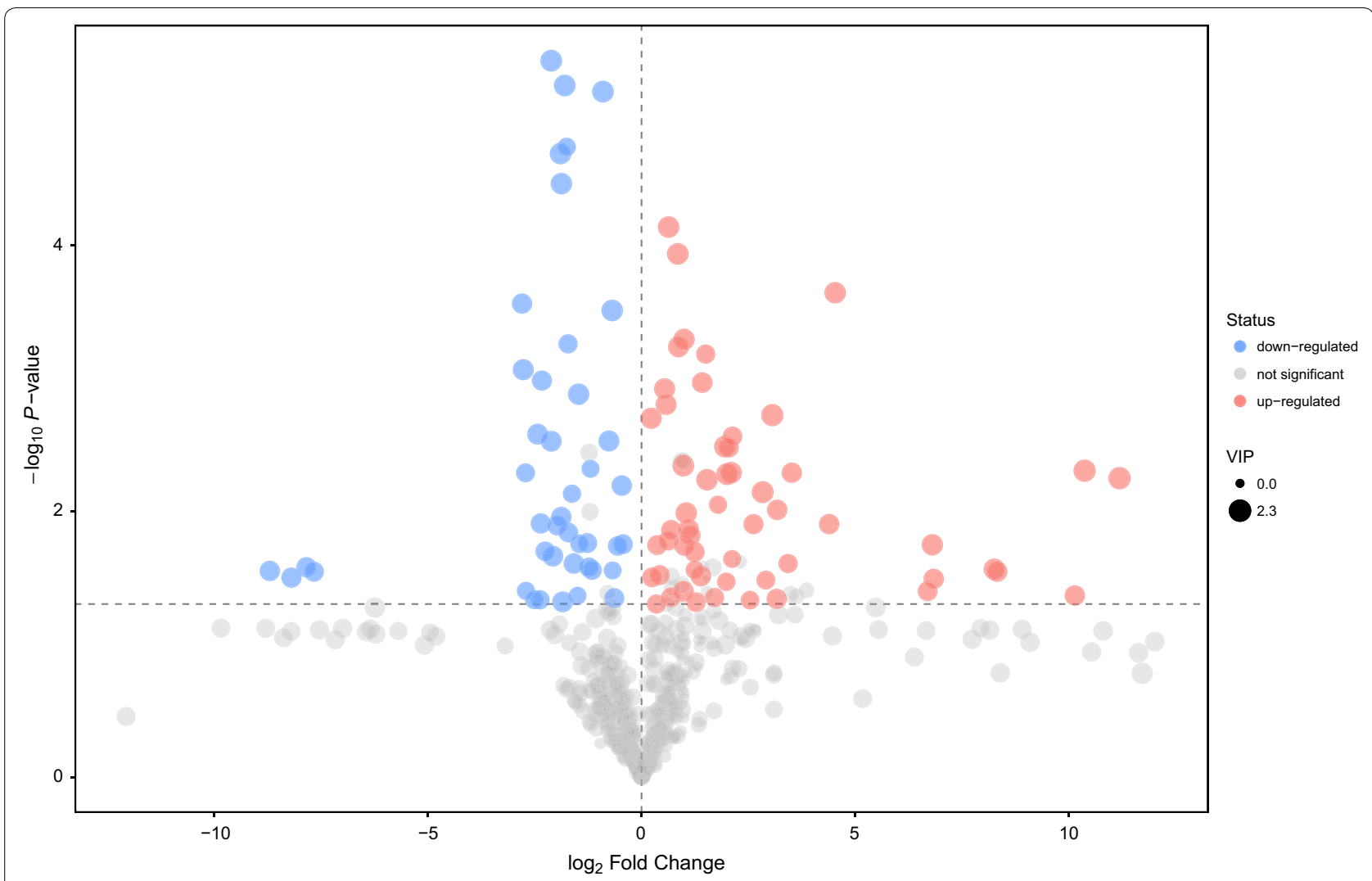

Fig. 3 Volcano plot for differential metabolites between control and plasma treatment group

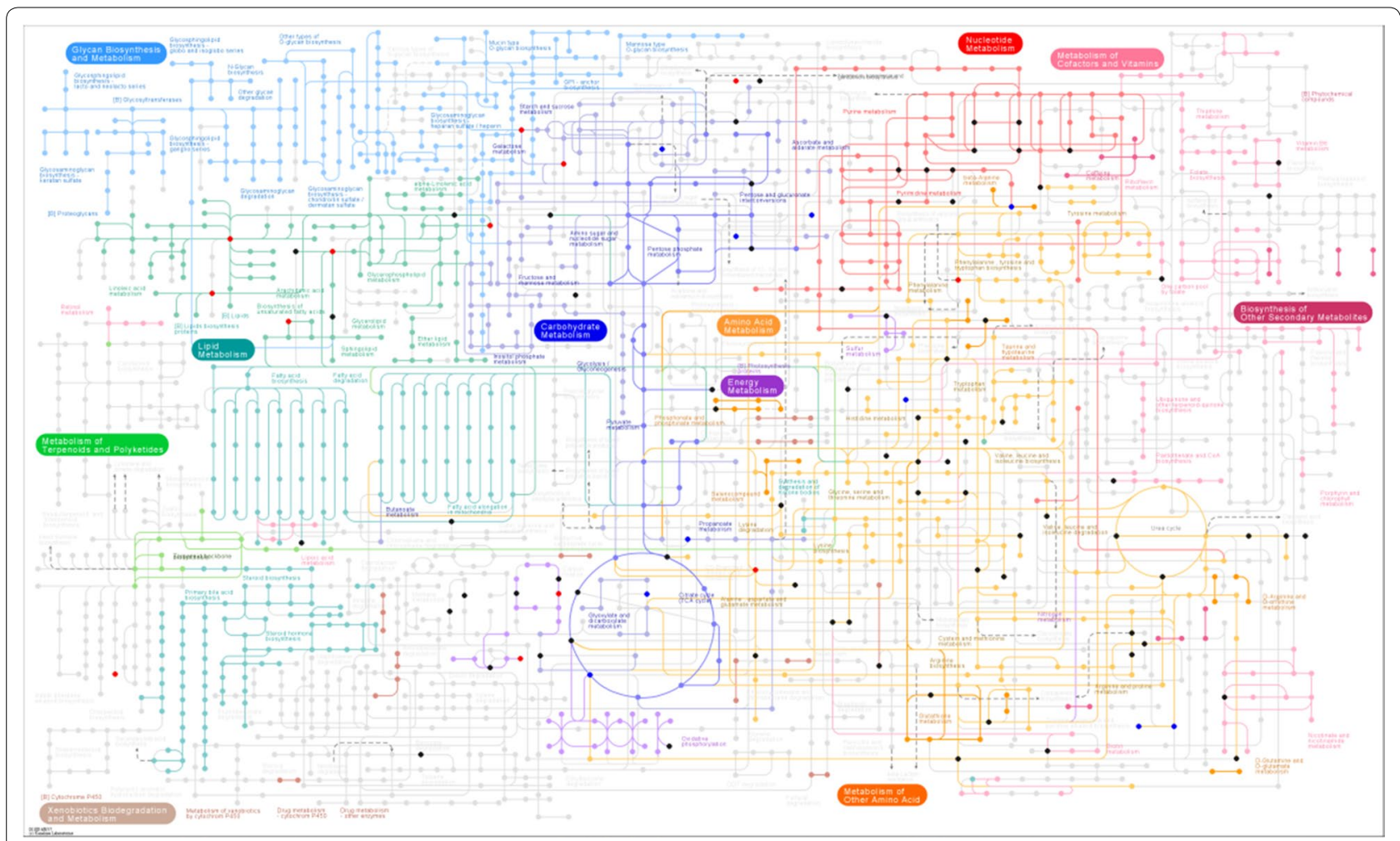

Fig. 4 Metabolic pathways with red/blue dots representing the differentially expressed compounds 


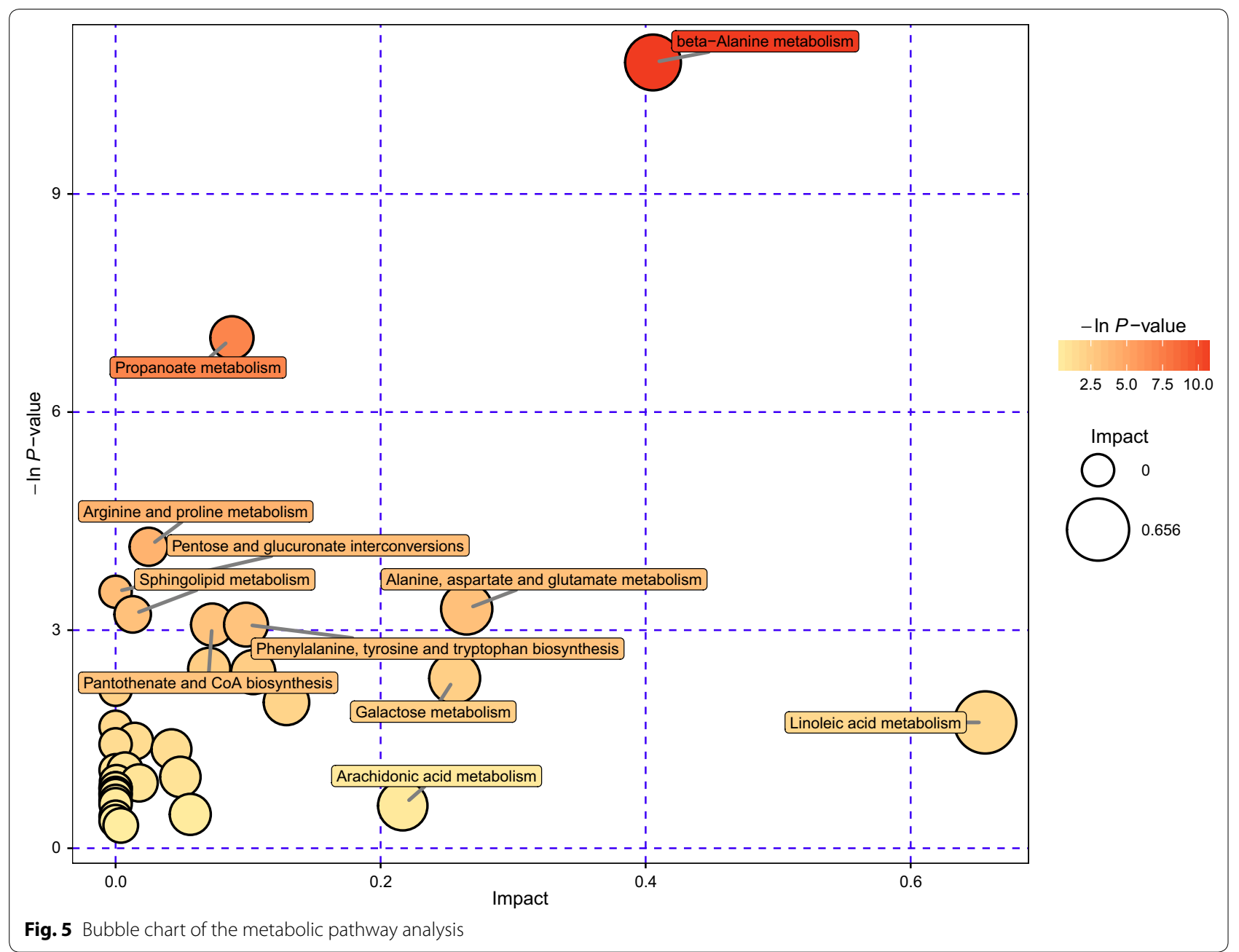

\section{Discussion}

Energy and material metabolism is the basic guarantee for cell survival. Adenosine triphosphate (ATP) is a currency in the cell that is used to store and deliver energy. In normal tissues, $90 \%$ of the ATP comes from oxidative phosphorylation, whereas only $10 \%$ comes from glycolysis [33]. In aerobic conditions, glycolysis is inhibited, known as the Pasteur effect. However, Warburg found that tumor cells are still prevalent with high rates of glucose uptake even under oxygenated conditions. The increased glycolytic metabolism and increased metabolites of lactate, which are ubiquitous in various tumor cells, called as the Warburg effect [34,35]. Although the efficiency of glycolysis is low, tumor cells can benefit from glycolysis: Firstly, Due to the rapid growth of tumor cells, there is a great demand for energy and more glycolytic production of ATP is required. Secondary, glycolysis intermediates such as 6-phosphate glucose, pyruvate can synthesize fatty acids, nucleic acids which are important for cell metabolism and biosynthesis. Therefore, the energy and material metabolism of tumor cells and normal cells are quite different. Atmospheric cold plasma, as a newly developed technology, can selectively induce tumor cell death. In addition, some related apoptosis pathway factors were reported although more mechanism need to be investigated. In our study, instead of study on the single apoptotic protein, we investigated the whole metabolism profiling to understand the effect of plasma on the metabolism of tumor cells. Because the metabolomic data typically contains a large number of variables that are interrelated, multivariate statistical methods such as PCA and OPLS-DA were used in this study [36]. We demonstrated the large scale metabolic profiling using GC-TOF mass spectrometry and found numerous significant differences between the gas control group and the plasma treatment group in myeloma tumor cells. By KEGG analysis of the metabolic pathways we found that beta-alanine metabolism pathway was the most significant changes after $\mathrm{He}$ gas plasma treatment in myeloma LP-1 cells. Alanine, beta-alanine and sarcosine share the same chemical formula $\mathrm{C}_{3} \mathrm{H}_{7} \mathrm{NO}_{2}$, but are 


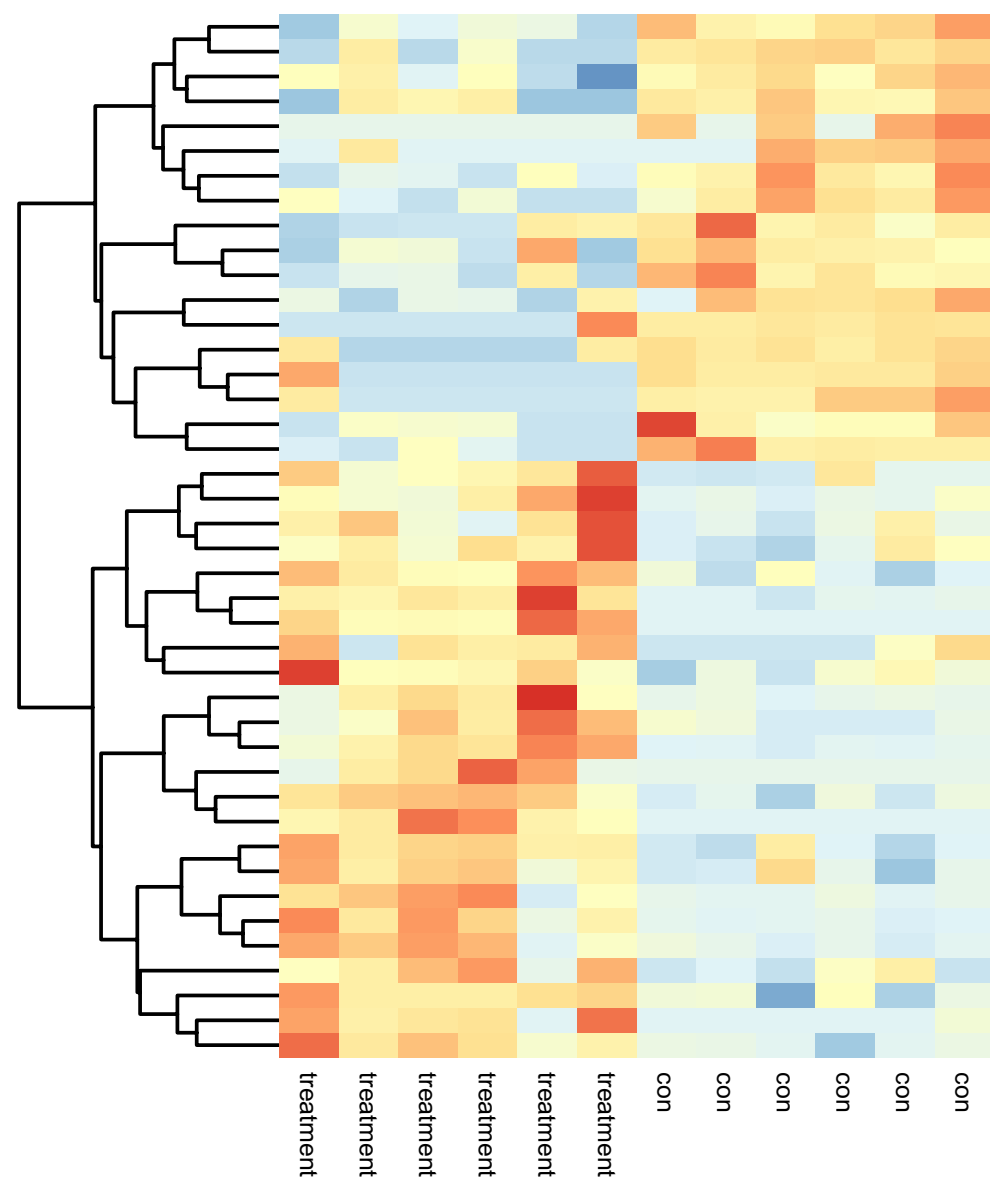

5-Methoxytryptamine 2

Nicotianamine

3-Hydroxypropionic acid 1

Allo-inositol

Tagatose 2

succinic acid

spermidine 1

spermine 4

2-hydroxybutanoic acid

beta-Alanine 1

oxalic acid

ribitol

putrescine 2

Phenaceturic acid 2

shikimic acid

4-Hydroxyquinoline

D-Arabitol

Sucrose-6-Phosphate

$\mathrm{N}$-Acetyl-D-galactosamine 1

Lyxose 1

galactose 2

$\mathrm{N}$-Ethylglycine 1

Pelargonic acid

D-(glycerol 1-phosphate)

phytosphingosine 2

lactose 2

hydroxyurea

aspartic acid 1

D-Fructose 1,6-bisphosphate 2

Pyrrole-2-Carboxylic Acid

Lignoceric acid

Monostearin

Digalacturonic acid 2

heptadecanoic acid

linoleic acid

alpha-Santonin 1

arachidonic acid

Abietic Acid 1

4-hydroxyph

4-hydroxyphenylpyruvate 1

Monoolein

O-Phosphorylethanolamine

androsterone 1

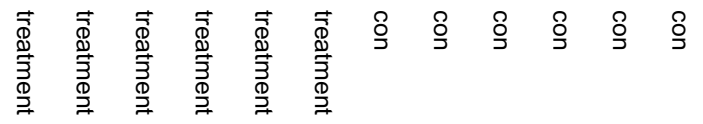

Fig. 6 Heatmap of hierarchical clustering analysis for plasma treatment and control group

structurally different. By GC-TOFMS analysis, beta-alanine is easy to separate from alanine and sarcosine duo to its distinct mass spectrum [37]. Beta-alanine is a direct precursor of pantothenic acid (PA) which is needed for the synthesis of coenzyme A $(\mathrm{CoA})$. In the tricarboxylic acid (TCA) cycle, CoA is important for pyruvate to enter as acetyl-CoA, and for $\alpha$-ketoglutarate to be transformed to succinyl-CoA [38]. In addition, CoA is involved in the biosynthesis of many important compounds such as fatty acids, cholesterol, and acetylcholine [39]. Therefore, by $\mathrm{He}$ plasma treatment, beta-alanine metabolism in myeloma tumor cells was suppressed, which disturbing the energy and material metabolism of the tumor cells and results in tumor cells death. Our data illustrated some details about the dysregulation of metabolism profiling by gas plasma for the first time. Although more researches need to be done to further analyze the mechanism under molecular microstructure, this study gives a general direction for further study. Meanwhile, more tumor cell lines and the treatment by different types of gas plasma devices will be done for metabolite profiling analysis, to further illustrate the biological effects in various tumor cells by different reactive species in gas plasma.

\section{Conclusions}

In conclusion, we demonstrated the effects of gas plasma on tumor cell metabolism by GC-TOF mass-spectrometry for the first time. By bioinformatics analysis we showed that plasma treatment could significantly alter the metabolite profiling of tumor cells. In addition, betaalanine metabolism pathway was the most susceptible to plasma treatment, which might be instructive to further detail the mechanism of biological effects induced by plasma treatment in tumor cells.

\section{Additional files}

Additional file 1: Table S1. KEGG metabolite mapping.

Additional file 2: Table S2. KEGG pathway.

Additional file 3: Table S3. Pathway metabolite mapping.

Additional file 4: Table S4. Pathway analysis. 


\section{Abbreviations}

MM: multiple myeloma; GC-TOF: gas-chromatography time-of-flight; CAP: cold atmospheric plasma; PCA: principal component analysis; VIP: variable importance in the projection; KEGG: kyoto encyclopedia of genes and genomes; ATP: adenosine triphosphate; PA: pantothenic acid; CoA: coenzyme A; TCA: tricarboxylic acid.

\section{Authors' contributions}

DHX and MGK contributed equally to this work, conceiving the study and writing the manuscript. YJX performed experiments and prepared the samples; NN analyzed the data and QJC participated in the experiment work; ZJL, XHW and DXL contributed to the visuals of this study. HLC provided assistance and revised this manuscript. All authors read and approved the final manuscript.

\section{Author details}

${ }^{1}$ State Key Laboratory of Electrical Insulation and Power Equipment, Centre for Plasma Biomedicine, Xi'an Jiaotong University, Xi'an 710049, Shaanxi, People's Republic of China. ${ }^{2}$ The School of Life Science and Technology, Xi'an Jiaotong University, Xi'an 710049, Shaanxi, People's Republic of China. ${ }^{3}$ Frank Reidy Center for Bioelectrics, Old Dominion University, Norfolk, VA 23508, USA. ${ }^{4}$ Department of Electrical and Computer Engineering, Old Dominion University, Norfolk, VA 23529, USA

\section{Acknowledgements}

Not applicable.

\section{Competing interests}

The authors declare that they have no competing interests.

\section{Availability of data and materials}

The datasets generated during and/or analyzed during the current study are available from the corresponding author on reasonable request.

\section{Consent for publication}

Not applicable.

\section{Ethics approval and consent to participate}

Not applicable.

\section{Funding}

This work is supported by the National Natural Science Foundation of China (Grant No. 51307135), China Postdoctoral Science Foundation (2017M610639), the Fundamental Research Funds for Central Universities and Special Fund of Shaanxi Postdoctoral Science Foundation (2017BSHTDZZ04).

\section{Publisher's Note}

Springer Nature remains neutral with regard to jurisdictional claims in published maps and institutional affiliations.

Received: 9 December 2017 Accepted: 14 March 2018

Published online: 20 March 2018

\section{References}

1. Laubach J, Richardson P, Anderson K. Multiple myeloma. Annu Rev Med. 2011;62:249-64.

2. Palumbo A, Anderson K. Multiple myeloma. N Engl J Med. 2011:364:1046-60.

3. Rajkumar SV, Kumar S. Multiple myeloma: diagnosis and treatment. Mayo Clin Proc. 2016:80:101-19.

4. Krishnan SR, Jaiswal R, Brown RD, Luk F, Bebawy M. Multiple myeloma and persistence of drug resistance in the age of novel drugs. Int J Oncol. 2016:49:33-50.

5. Podar K, Chauhan D, Anderson KC. Bone marrow microenvironment and the identification of new targets for myeloma therapy. Leukemia. 2009;23:10-24.
6. Armoiry X, Tsertsvadze A, Connock M, Melendez-Torres G, Clarke A. Systematic review and network meta-analysis of treatment outcomes for multiple myeloma. J Clin Oncol. 2017;35:2975-6.

7. Malard F, Harousseau J, Mohty M. Multiple myeloma treatment at relapse after autologous stem cell transplantation: a practical analysis. Cancer Treat Rev. 2017;52:41-7

8. Graves DB. Reactive species from cold atmospheric plasma: implications for cancer therapy. Plasma Process Polym. 2014;11:1120-7.

9. Keidar M, Shashurin A, Volotskova O, et al. Cold atmospheric plasma in cancer therapy. Phys Plasmas. 2013;20:057101.

10. Kvam E, Davis B, Mondello F, Garner AL. Nonthermal atmospheric plasma rapidly disinfects multidrug-resistant microbes by inducing cell surface damage. Antimicrob Agents chemother. 2012;56:2028-36.

11. Gurol C, Ekinci FY, Aslan N, Korachi M. Low temperature plasma for decontamination of E. coli in milk. Int J Food Microbiol. 2012;157:1-5.

12. Keidar M, Walk R, Shashurin A, et al. Cold plasma selectivity and the possibility of a paradigm shift in cancer therapy. $\mathrm{Br} J$ Cancer 2011:105:1295-301.

13. Isbary G, Morfill G, Schmidt HU, et al. A first prospective randomized controlled trial to decrease bacterial load using cold atmospheric argon plasma on chronic wounds in patients. Br J Dermatol. 2010;163:78-82.

14. Ishaq M, Evans M, Ostrikov K. Effect of atmospheric gas plasmas on cancer cell signaling. Int J Cancer. 2014;134:1517-28.

15. Kalghatgi SU, Fridman G, Cooper M, et al. Mechanism of blood coagulation by nonthermal atmospheric pressure dielectric barrier discharge plasma. IEEE Trans Plasma Sci. 2007;35:1559-66.

16. Fridman G, Friedman G, Gutsol A, et al. Applied plasma medicine. Plasma Process Polym. 2008;5:503-33.

17. Xu D, Wang B, Xu Y, et al. Intracellular ROS mediates gas plasma-facilitated cellular transfection in 2D and 3D cultures. Sci Rep. 2016;6:27872.

18. Zhang X, Li M, Zhou R, Feng K, Yang S. Ablation of liver cancer cells in vitro by a plasma needle. Appl Phys Lett. 2008;93:021502.

19. Thiyagarajan M, Sarani A, Gonzales XF. Characterization of an atmospheric pressure plasma jet and its applications for disinfection and cancer treatment. Stud Health Technol Inform. 2013;184:443-9.

20. Kim CH, Kwon S, Bahn JH, et al. Effects of atmospheric nonthermal plasma on invasion of colorectal cancer cells. Appl Phys Lett. 2010:96:243701-3.

21. Yan X, Zou F, Zhao S, et al. On the mechanism of plasma inducing cel apoptosis. IEEE Trans Plasma Sci. 2010:38:2451-7.

22. Vandamme M, Robert E, Pesnel S, et al. Antitumor effect of plasma treatment on U87 glioma xenografts: preliminary results. Plasma Process Polym. 2010;38:748-57

23. Brulle L, Vandamme M, Ries $D$, et al. Effects of a non thermal plasma treatment alone or in combination with gemcitabine in a MIA PaCa2-luc orthotopic pancreatic carcinoma model. PLoS ONE. 2012;7:e52653.

24. Ahn HJ, Kim Kl, Kim G, et al. Atmospheric-pressure plasma jet induces apoptosis involving mitochondria via generation of free radicals. PLoS ONE. 2011:6:e28154.

25. Majumdar A, Ummanni R, Schroder K, Walther R, Hippler R. Cancer cells (MCF-7, Colo-357, and LNCaP) viability on amorphous hydrogenated carbon nitride film deposited by dielectric barrier discharge plasma. J Appl Phys. 2009:106:034702.

26. Min Joh H, Ja Kim S, Chung T, Leem S. Reactive oxygen species-related plasma effects on the apoptosis of human bladder cancer cells in atmospheric pressure pulsed plasma jets. Appl Phys Lett. 2012;101:053703.

27. Walk RM, Snyder JA, Srinivasan P, et al. Cold atmospheric plasma for the ablative treatment of neuroblastoma. J Pediatr Surg. 2013:48:67-73.

28. Xu D, Luo X, Xu Y, et al. The effects of cold atmospheric plasma on cell adhesion, differentiation, migration, apoptosis and drug sensitivity of multiple myeloma. Biochem Biophys Res Commun. 2016;473:1125-32.

29. Kroemer G, Pouyssegur J. Tumor cell metabolism: cancer's Achilles' heel. Cancer Cell. 2008;13:472-82.

30. Cairns RA, Harris IS, MakTW. Regulation of cancer cell metabolism. Nat Rev Cancer. 2011;11:85-95.

31. DeBerardinis RJ, Sayed N, Ditsworth D, Thompson CB. Brick by brick: metabolism and tumor cell growth. Curr Opin Genet Dev. 2008;18:54-61.

32. Xu D, Liu D, Wang $\mathrm{B}$, et al. In situ $\mathrm{OH}$ generation from $\mathrm{O}_{2}{ }^{-}$and $\mathrm{H}_{2} \mathrm{O}_{2}$ plays a critical role in plasma-induced cell death. PLoS ONE. 2015;10:e0128205. 
33. Mayevsky A. Mitochondrial function and energy metabolism in cancer cells: past overview and future perspectives. Mitochondrion. 2009;9:165-79.

34. Vander Heiden MG, Cantley LC, Thompson CB. Understanding the Warburg effect: the metabolic requirements of cell proliferation. Science. 2009;324:1029-33.

35. DeBerardinis RJ. Is cancer a disease of abnormal cellular metabolism? New angles on an old idea. Genet Med. 2008;10:767-77.

36. Bylesjö M, Rantalainen $\mathrm{M}, \mathrm{Cloarec} \mathrm{O}$, et al. OPLS discriminant analysis: combining the strengths of PLS-DA and SIMCA classification. J Chemom. 2006:20:341-51.
37. Meyer TE, Fox SD, Issaq HJ, et al. A reproducible and high-throughput HPLC/MS method to separate sarcosine from a-and $\beta$-alanine and to quantify sarcosine in human serum and urine. Anal Chem. 2011;83:5735-40

38. Fernie AR, Carrari F, Sweetlove LJ. Respiratory metabolism: glycolysis, the TCA cycle and mitochondrial electron transport. Curr Opin Plant Biol. 2004;7:254-61.

39. Hutschenreuther A, Birkenmeier G, Bigl M, Krohn K, Birkemeyer C. Glycerophosphoglycerol, beta-alanine, and pantothenic acid as metabolic companions of glycolytic activity and cell migration in breast cancer cell lines. Metabolites. 2013;3:1084-101.

\section{Submit your next manuscript to BioMed Central and we will help you at every step:}

- We accept pre-submission inquiries

- Our selector tool helps you to find the most relevant journal

- We provide round the clock customer support

- Convenient online submission

- Thorough peer review

- Inclusion in PubMed and all major indexing services

- Maximum visibility for your research

Submit your manuscript at www.biomedcentral com/submit 